\title{
Application of Fuzzy Logic Algorithm to Single-Molecule Force Spectroscopy of the Streptavidin-Biotin System
}

\author{
Hiroyuki Tahara ${ }^{*}$, Takashi Nyu ${ }^{1 *}$, Evan Angelo Quimada Mondarte1, Tatsuhiro Maekawa1, \\ Tomohiro Hayashi'1,2\#
}

${ }^{1}$ Tokyo Institute of Technology, Department of Materials Science and Engineering, School of Materials and Chemical Technology, Yokohama, Japan ${ }^{2}$ JST-PRESTO, Kawaguchi-shi, Saitama, Japan

Email: "hayashi.t.al@m.titech.ac.jp

How to cite this paper: Tahara, H., Nyu, T., Mondarte, E.A.Q., Maekawa, T. and Hayashi, T. (2018) Application of Fuzzy Logic Algorithm to Single-Molecule Force Spectroscopy of the Streptavidin-Biotin System. Advances in Materials Physics and Chemistry, 8, 217-226.

https://doi.org/10.4236/ampc.2018.85014

Received: April 5, 2018

Accepted: May 26, 2018

Published: May 29, 2018

Copyright $\odot 2018$ by authors and Scientific Research Publishing Inc. This work is licensed under the Creative Commons Attribution International License (CC BY 4.0)

http://creativecommons.org/licenses/by/4.0/

\begin{abstract}
The rupture force of the streptavidin-biotin complex was investigated using atomic force microscopy (AFM). The most frequently observed rupture force (MFOF), which is essential for the evaluation of the potential landscape, was evaluated by processing 22,500 force curves using two methods. One method is a conventional method, which is usually built in commercial AFM systems, i.e., difference between the baseline value and the minimum force value in the force curve. The other is a detection of rupture events based on a fuzzy logic algorithm to detect the rupture event from analyzing the shape of the force curves. Our statistical analysis revealed that the conventional method exhibited a significant artifact, which is the increase in the population of small forces comparable to thermal noise of cantilevers, resulting in a smaller MFOF. Based on this finding, we discuss the choice of a method and its effecton the illustrated potential landscapes of ligand-receptor complexes.
\end{abstract}

\section{Keywords}

Single-Molecule Force Spectroscopy, Atomic Force Microscopy, Fuzzy Logic Algorithm, Streptavidin, Biotin

\section{Introduction}

Single-molecule force spectroscopy (SMFS) is a promising technique to investigate the nature of inter-or intra-molecular interactions and the mechanical properties of various molecular systems (ligand-receptor systems, protein molecules, polymer chains, etc.) from their mechanical responses. Common SMFS ${ }^{*}$ Equally contributed authors. 
tools are atomic force microscopy (AFM), blood cell force spectroscopy, and optical or magnetic tweezers [1] [2]. By measuring force along the reaction coordinate (direction of pulling) and analyzing the forces, we can reveal the number of and positions of internal potential barriers. The successful examples range over the potential landscapes of binding of ligand-receptor complexes [3] [4], protein-unfolding and refolding pathways [5] [6] [7], and other inter and intramolecular interactions [8] [9] [10] [11] [12].

For the above applications, we need to analyze a large number (at least hundreds) of force-separation curves and to perform statistical analysis of the observed rupture forces in the curves. By measuring the rupture forces at different loading rates and applying the Bell-Evans theory (Equation (1)) [13] [14], we can illustrate the overview of the potential landscape. The theory is expressed in

$$
f^{*}=k_{B} T / x_{b}\left\{\ln r_{l}+\ln \left(\tau_{0} x_{b} / k_{B} T\right)\right\},
$$

where $f^{*}, k_{B}, T, x_{b}, r_{b}$ and $\tau_{0}$ are the most frequently observed rupture force (MFOF), Boltzmann constant, temperature, the position of a potential barrier, loading rate, and natural bond life time, respectively. To evaluate MFOF from a histogram of rupture forces of a single ligand-receptor complex, we need to collect the force curves with the single-molecule event preferentially. For this, the densities of ligand and receptor molecules on the probe and substrate are optimized (lowered) for the preferential formation of the single bond. With the optimization, the probability of observing rupture events is $10 \%-20 \%$. Therefore, construction of a histogram containing 100 data points requires measurements of around $500-1000$ force-separation curves. One can easily imagine that the manual detection of interaction force from each force-separation curve is practically impossible.

A conventional detection way, which is usually built in commercial AFM systems, is through the subtraction of the minimum value from the baseline of the curve. This method evaluates any adhesion force operating between the tip and surface, including non-specific interaction and thermal noises together with the specific ligand-receptor interaction. Therefore, we have to set a filter to carefully isolate forces coming from specific interaction for data analysis. In common experimental configurations, the ligand (or receptor) is immobilized on a tip or surface via a flexible polymer chain (in most cases polyethylene glycol). Therefore, most of the cases, rupturing events of ligand-receptor bonds appear after the stretch of the polymer (Figure 1). The characteristic force-separation profile is well fitted to the worm-like-chain or free-joint-chain models [15] [16] [17].

There are several methods of detecting the polymer stretching, e.g., fitting of the stretch curves [18] and evaluation of the shape of curves at the rupture event [19]. Among them, Kasas et al. proposed a strong method that enables semi-automated detection of rupturing event of specific interaction after the stretching of a polymer chain [20]. This method is successfully utilized by several groups [21]-[33]. However, in most of the reports, the analyses of the force curves were still performed without the above detecting techniques. 


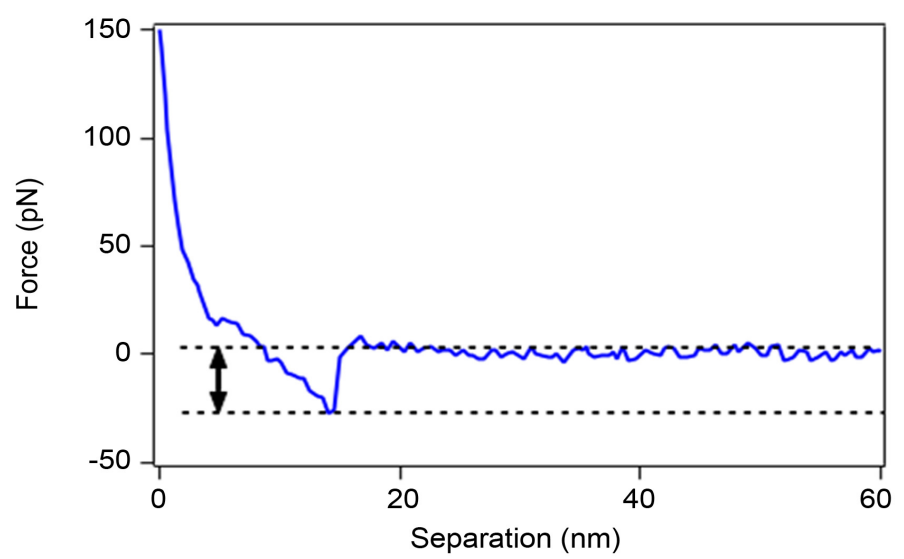

(a)

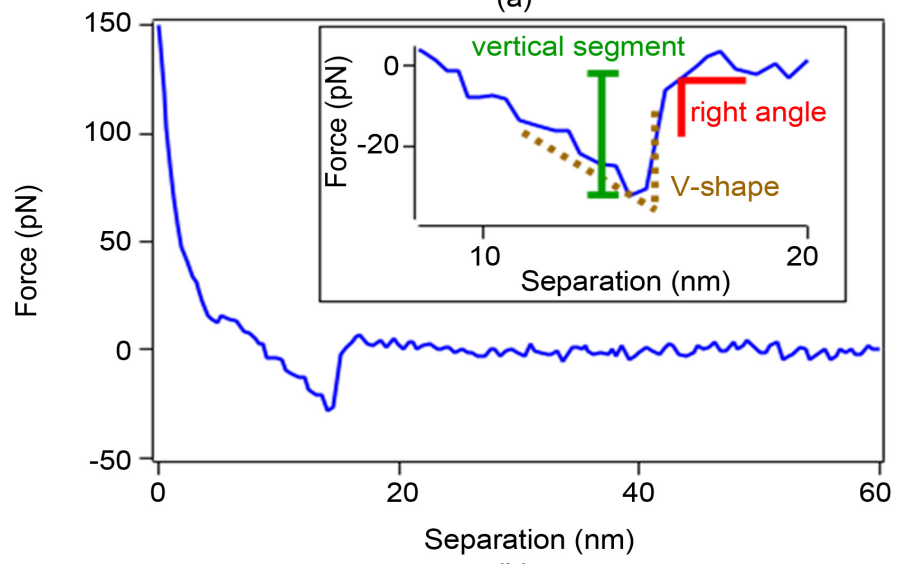

(b)

Figure 1. Typical force-separation curve and detections of a rupture event (a) by the conventional method, in which the difference between the minimum force value from and the baseline was determined and (b) by fuzzy logic algorithm in which three criteria (V-shape, vertical steep segment, and right angle) were utilized.

In this work, we discuss the difference in histograms of rupture forces obtained by the conventional approach and the method developed by Kasas et al., in particular, the artifacts of the conventional method appearing in the histogram of force and its origin in detail, which has not been discussed so far.

\section{Experimental}

\subsection{Preparation of Substrates}

We immobilized streptavidin molecules to self-assembled monolayers (SAMs) of alkanethiols on Au (Furuya Meatal, Japan)/Ge (The Nilaco Corporation, Japan)/Si (Furuuchi Chemical, Japan) substrates. The metal substrates were fabricated by thermal evaporation under a vacuum (base pressure is around $2 \times 10^{-6}$ $\mathrm{Pa}$ ). To control the density of streptavidin molecules on the substrate, we fabricated the SAM from two precursor thiol molecules with different terminal groups: $\mathrm{HSC}_{11}(\mathrm{EG})_{6} \mathrm{OCH}_{2} \mathrm{COOH}$ (MW: $526.73 \mathrm{~g} / \mathrm{mol}$, ProChimia Surfaces, Poland) and $\mathrm{HSC}_{11}(\mathrm{EG})_{3} \mathrm{OH}$ (MW: $336.53 \mathrm{~g} / \mathrm{mol}$, ProChimia Surfaces, Poland). A 
thiol solution $(1 \mathrm{mM})$ was prepared by dissolving $\mathrm{HSC}_{11}(\mathrm{EG})_{6} \mathrm{OCH}_{2} \mathrm{COOH}$ : $\mathrm{HSC}_{11}(\mathrm{EG})_{3} \mathrm{OH}$ in ethanol (5 mL, Wako, Japan) at a molar ratio of 1:3. Next, we activated the $-\mathrm{COOH}$ terminal group to form a amide bond with $-\mathrm{NH}_{2}$ groups of streptavidin. The substrates were then transferred to a solution of 1-ethyl-3-(3-dimethylaminopropyl)carbodiimide hydrochloride (EDC, Tokyo Chemical Industry, Japan) (5 mL and $75 \mathrm{mM}$ ) and $300 \mathrm{mM} N$-hydroxysuccinimide (NHS, KANTO CHEMICAL, Japan) in Milli-Q water and reacted for $30 \mathrm{~min}$. Finally, the substrates with activated groups were then immersed in the streptavidin (Sigma-Aldrich, USA) in phosphate-buffered silane (PBS, Sigma-Aldrich, USA) for $2 \mathrm{~h}$ to complete the immobilization of streptavidin molecules.

\subsection{Immobilization of Biotin Molecules on AFM Probes}

A solution $(80 \mu \mathrm{L})$ of (3-aminopropyl)triethoxysilane (APTES, Tokyo Chemical Industry, Japan) and triethylamine (TEA, Wako, Japan) at a volume ratio of 3:1 was prepared under in dry nitrogen. APTES aids to the silane coupling of the amino acid to the tip via a linker, while TEA catalyzes the reaction. The probes were placed in a sealed box together with the APTES/TEA solution allowing the vapor molecules to attach to the tip surface (silicon) for $3 \mathrm{~h}$ in nitrogen atmosphere producing a primary amine terminal group extending from the tip surface. $\alpha$-Biotin-(ethylene glycol) $)_{24}-\omega$-succinimidyl propionate (Biotin- $\mathrm{dPEG}_{24}-\mathrm{NHS}$, Quanta BioDesign, USA) and $\alpha$-Methoxy-(ethylene glycol) $)_{24}-\omega$-propionic acid succinimidyl ester (MeO-dPEG ${ }_{24}$-NHS, Quanta BioDesign, USA) were dissolved in PBS $(1 \mathrm{mM})$. The probes were then immersed in the mixture of Biotin- $\mathrm{dPEG}_{24}-\mathrm{NHS}$ and $\mathrm{MeO}-\mathrm{dPEG}_{24}-\mathrm{NHS}$ at a volume ratio of $1: 5$ for $12 \mathrm{~h}$.

\subsection{Atomic Force Microscopy and the Measurement Conditions}

We employed a commercial AFM system (MFP-3D Bio, Oxford Instruments, USA) equipped with a liquid cell. All measurements were performed in PBS at room temperature $(300 \mathrm{~K})$. The spring constants of cantilevers were calibrated by measuring the thermal noise [34]. The triggering force and loading rate were fixed at $150 \mathrm{pN}$ and $126 \mathrm{pN} / \mathrm{sec}$, respectively. The deflection sensitivity was evaluated from the force-separation curve in the linear compliance region (around under $1 \mathrm{nN}$ ), where the force increases linearly as a function of piezo displacement, after all measurements to avoid mechanical damage to the probe. We used a cantilever (Biolever mini, Olympus, Japan) whose nominal spring constant was $60 \mathrm{pN} / \mathrm{nm}$.

\section{Data Analysis}

\subsection{Conversion of Force-Displacement Curves to Force-Separation Curves}

First, force-piezo displacement curves were converted to force-separation curves by using the obtained deflection sensitivity [35]. In this work, the zero separation was defined by the $x$-positions at the triggering force of $150 \mathrm{pN}$. 


\subsection{Detection of Streptavidin-Biotin Interaction from Force-Separation Curves}

In this work, we employed two methods to extract the force of streptavidin-biotin interaction from force-separation curves. One is a subtraction of the minimum value in the force curve from the baseline [Figure 1(a)]. The base line was defined by the average of 10 points at the separation of $60 \mathrm{~nm}$ in the curve.

The other approach was a method based on fuzzy logic algorithm developed by the Kasas et al. In this approach, the events of the rupture of the streptavidin-biotin bond was detected with the shape of the peak. The detection was based on three criteria, which were a V-shape, vertical steep segment, and right angle [Figure 1(b)]. Three vectors to calculate scores were set same as the parameters used by Kasas et al. We eliminated that force curves with multiple rupture events, because the loading force was shared by multiple bonds resulting in a lowering of the loading rate. Considering the population of such curves (less than $1 \%$ ), the effect on the overall statistics is negligible.

Considering our design of the tip [biotin immobilized via $10 \mathrm{~nm}$ of PEG chain and streptavidin (around $5 \mathrm{~nm}$ ) fixed on the surface via $2 \mathrm{~nm}$ of PEG chain], rupture events with longer rupture separation may involve other molecular events such as unfolding of streptavidin, stretching of contaminants. Therefore, we discuss the rupture events with rupture separations shorter than $30 \mathrm{~nm}$ for the both cases.

\section{Results \& Discussion}

Our analysis using the fuzzy logic algorithm method revealed that the rupture events were detected in only $11 \%$ of the total force curves. The MFOF was observed at $27 \mathrm{pN}$ [Figure 2(a)], in agreement with the previously reported results [13], indicating that single-molecule events were dominant, and that there is a small contribution of events for rupturing of double or higher multiple bonds to the total statistic.

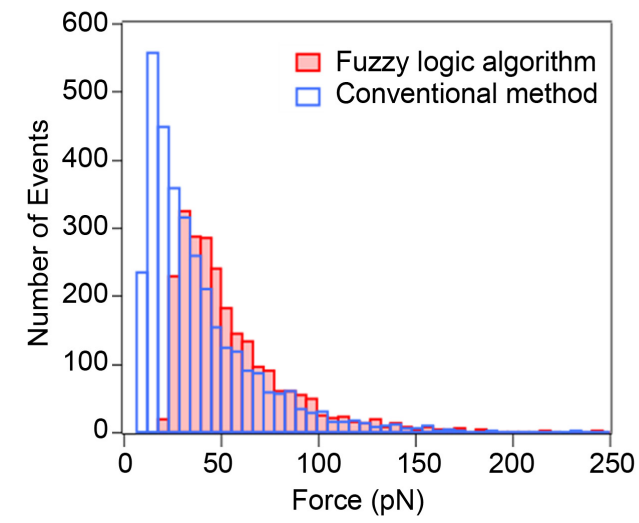

(a)

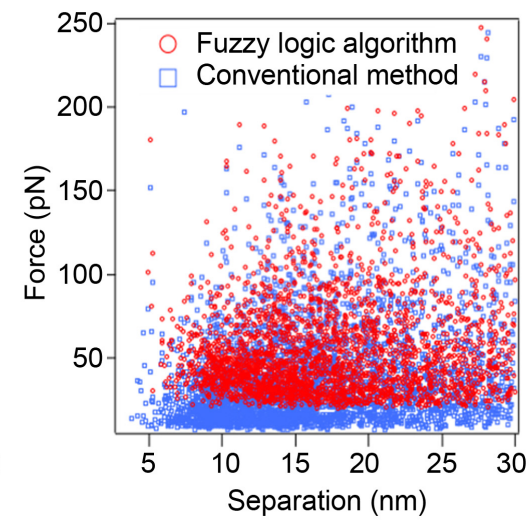

(b)

Figure 2. Histograms of (a) force and (b) distribution of all detected rupture events by conventional and fuzzy logic algorithm methods. 
The histograms clearly exhibit the difference in the statistics of rupture forces [Figure 2(a)]. The MFOF values are significantly different, i.e., 10 and $27 \mathrm{pN}$ from the conventional and fuzzy logic algorithm methods, respectively. The overall comparison is shown in Figure 2(b) revealed that the false detection of rupture event in weak rupture force region $(<15 \mathrm{pN})$ distributes in the overall range of the rupture separation.

The conventional method returns one "rupturing" force value from each force-separation curve even without any rupturing event. It picked up the minimum value even from the noise, generating the increase in the number of rupture force comparable to the magnitude of the thermal noise as shown in Figure 3(a) (in this work around $10 \mathrm{pN}$ in peak-to-peak). These results show in the increase of the population in the region of small rupture forces [Figure 2(a)].

Next, we discuss several examples of false detections that were avoided by the fuzzy logic algorithm. Figure 3(a) exhibits a typical false detection of thermal noise of cantilever from force curves without any rupture events. The fuzzy logic algorithm perfectly succeeded in eliminating this kind of data from the statistics. As to the case of Figure 3(b), the stretching of contaminants seemed confined between the tip and surface. In this case, a strong adhesion with a long rupture separation was observed. The long-separation peeling-off process exhibited a long plateau shape and dull peaks before the rupture in the negative force. The fuzzy logic algorithm rejected similar cases because of the low scores for the V-shape.

Finally, we discuss the artifact on the potential landscape evaluated based on the Bell-Evans theory. As we discussed, the conventional method tends to overestimate the population of weak rupture force by picking up the thermal noise, resulting in the decrease in the MFOF. The overall change in the force-loading rate profile, which is used to estimate the $x_{b}$ and $\tau_{0}$, depends on the combination of ligands and receptors and the design of the tip and surface. Here, we display two examples of the artifact of the conventional method on plots of

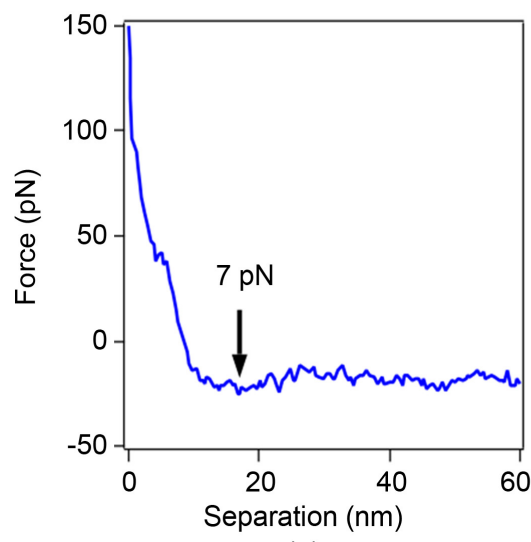

(a)

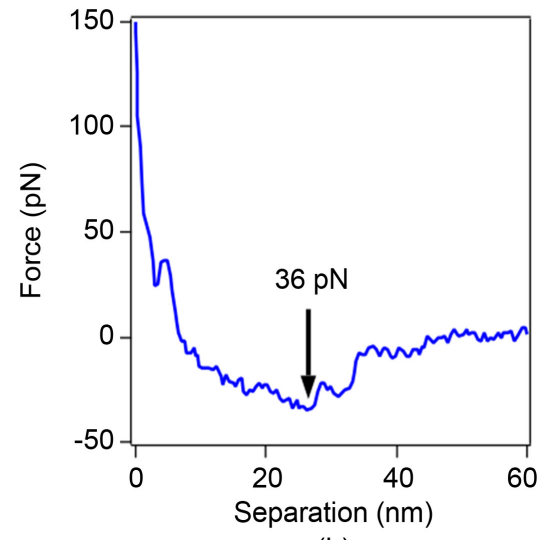

(b)

Figure 3. Examples of false-detected rupture events by the conventional method (a) thermal noise, (b) non-specific interaction probably due to hydrophobic contaminant confined between the tip and surface. 
force-loading rate and potential landscapes (Figure 4). The main artifact, an overall lowering of the rupture force in every loading rate results in the shorter $\tau_{0}$ (the inverse of the intersection of the plot and the $\mathrm{x}$-axis), indicating the lower potential barrier than the real barrier height. Even small changes in a slope of the plot lead to the change in both in $x_{b}$ and in particular $\tau_{0}$ [Equation (1)], since $\mathrm{x}$-axis is in a logarithmic scale and the position of the intersection drastically changes.

\section{Conclusion}

We investigated statistics of single-molecule interaction forces of the streptavidin-biotin ligand-receptor system. To effectively extract single molecular events from 22,500 force-separation curves obtained from AFM measurements, we employed two different ways. One is a conventional way that is built in major commercial AFM systems. It calculates the difference between the minimum value in the curve and baseline. The other is a method based on fuzzy logic algorithm initially proposed by Kasas et al. In this method, the rupture events in the force-separation curves were recognized by the scoring of characteristic shape. We found that the obvious difference in the obtained histograms of the rupture force. The conventional way frequently exhibited false detection, which picked up the thermal noise as a rupturing event. This false detection contributed to the increase in the population of the force weaker than $20 \mathrm{pN}$, resulting in the lowering of the most frequently observed rupture force, which is an important factor to illustrate potential landscapes of ligand-receptor systems based on the Bell-Evans theory. The conventional way also undesirably detected the non-specific interaction as the rupturing event. We found that this also provides undesirable effects on histograms in an overall range. Therefore, we concluded that the effective selection of force curves for data analyses using approaches of shape recognition is important for the quantitative analyses of various force measurements (adhesion, deformation, protein-stretching, etc.).

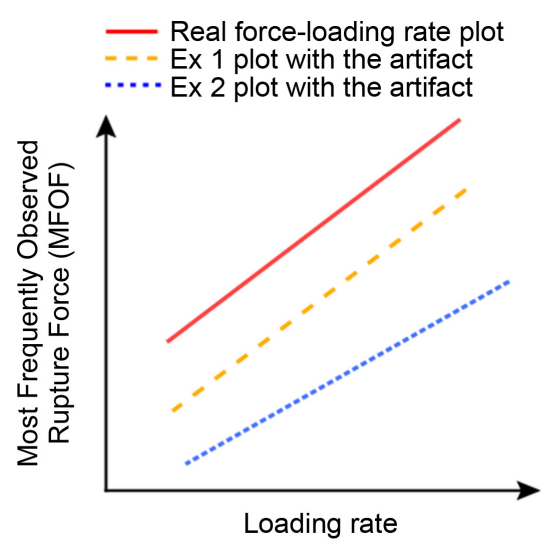

(a)

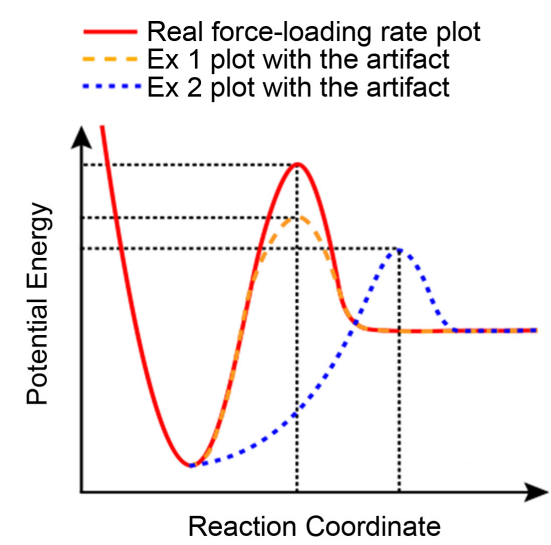

(b)

Figure 4. Schematic illustrations of the artifact on (a) force-loading rate plots (note that $\mathrm{x}$-axis is in a logarithmic scale) and (b) the resulting potential landscapes evaluated by the Bell-Evans theory. 


\section{Acknowledgements}

A part of this work was performed under the Cooperative Research Program of "NJRC Mater. \& Dev." This work is also supported by JST-PRESTO and MEXT KAKENHI (17K20095 and 15KK0184).

\section{References}

[1] Miller, H., Zhou, Z., Shepherd, J., Wollman, A.J.M. and Leake, M.C. (2018) Single-Molecule Techniques in Biophysics: A Review of the Progress in Methods and Applications. Reports on Progress in Physics, 81, Article ID: 024601. https://doi.org/10.1088/1361-6633/aa8a02

[2] Guttenberg, Z., Bausch, A.R., Hu, B., Bruinsma, R., Moroder, L. and Sackmann, E. (2000) Measuring Ligand-Receptor Unbinding Forces with Magnetic Beads: Molecular Leverage. Langmuir, 16, 8984-8993. https://doi.org/10.1021/la000279x

[3] Zhang, X. and Moy, V.T. (2003) Cooperative Adhesion of Ligand-Receptor Bonds, Biophysical Chemistry, 104, 271-278. https://doi.org/10.1016/S0301-4622(02)00381-2

[4] Vera, A.M. and Carrion-Vazquez, M. (2016) Direct Identification of Protein-Protein Interactions by Single-Molecule Force Spectroscopy. Angewandte Chemie International Edition, 55, 13970-13973. https://doi.org/10.1002/anie.201605284

[5] Rief, M., Gautel, M., Oesterhelt, F., Fernandez, J.M. and Gaub, H.E. (1997) Reversible Unfolding of Individual Titin Immunoglobulin Domains by AFM. Science, 276, 1109-1112. https://doi.org/10.1126/science.276.5315.1109

[6] Berkemeier, F., Bertz, M., Xiao, S.B., Pinotsis, N., Wilmanns, M., Grater, F. and Rief, M. (2011) Fast-Folding $\alpha$-Helices as Reversible Strain Absorbers in the Muscle Protein Myomesin. Proceedings of the National Academy of Sciences of the United States of America, 108, 14139-14144. https://doi.org/10.1073/pnas.1105734108

[7] Stigler, J. and Rief, M. (2015) Ligand-Induced Changes of the Apparent Transition-State Position in Mechanical Protein Unfolding. Biophysical Journal, 109, 365-372. https://doi.org/10.1016/j.bpj.2015.06.009

[8] Hanhijarvi, K.J., Ziedaite, G., Bamford, D.H., Haeggstrom, E. and Poranen, M.M. (2017) Single-Molecule Measurements of Viral ssRNA Packaging. RNA, 23, 119-129. https://doi.org/10.1261/rna.057471.116

[9] Bergues-Pupo, A.E., Gutierrez, I., Arias-Gonzalez, J.R., Falo, F. and Fiasconaro, A. (2017) Mesoscopic Model for DNA G-Quadruplex Unfolding. Scientific Reports, 7, Article No. 11756. https://doi.org/10.1038/s41598-017-10849-2

[10] Zhang, B., Zheng, W., Papoian, G.A. and Wolynes, P.G. (2016) Exploring the Free Energy Landscape of Nucleosomes. Journal of the American Chemical Society, 138 8126-8133. https://doi.org/10.1021/jacs.6b02893

[11] Nam, G.M. and Makarov, D.E. (2016) Extracting Intrinsic Dynamic Parameters of Biomolecular Folding from Single-Molecule Force Spectroscopy Experiment. Protein Science, 25, 123-134. https://doi.org/10.1002/pro.2727

[12] Arai, Y., Okabe, K., Sekiguchi, H., Hayashi, T. and Hara, M. (2011) Nanoscale Chemical Composition Analysis Using Peptides Targeting Inorganic Materials. Langmuir, 27, 2478-2483. https://doi.org/10.1021/la104178h

[13] Merkel, R., Nassoy, P., Leung, A., Ritchie, K. and Evans, E. (1999) Energy Landscapes of Receptor-Ligand Bonds Explored with Dynamic Force Spectroscopy. Nature, 397, 50-53. https://doi.org/10.1038/16219 
[14] Evans, E.B. (1999) Looking inside Molecular Bonds at Biological Interfaces with Dynamic Force Spectroscopy. Biophysical Chemistry, 82, 83-97. https://doi.org/10.1016/S0301-4622(99)00108-8

[15] Janshoff, A., Neitzert, M., Oberdorfer, Y. and Fuchs, H. (2000) Force Spectroscopy of Molecular Systems-Single Molecule Spectroscopy of Polymers and Biomolecules. Angewandte Chemie International Edition, 39, 3212-3237. https://doi.org/10.1002/1521-3773(20000915)39:18<3212::AID-ANIE3212>3.0.CO;2 $-\mathrm{X}$

[16] Oesterhelt, F., Rief, M. and Gaub, H.E. (1999) Single Molecule Force Spectroscopy by AFM Indicates Helical Structure of Poly(Ethylene-Glycol) in Water. New Journal of Physics, 1, 1998-1999.

[17] Zhang, W.K., Zou, S., Wang, C. and Zhang, X. (2000) Single Polymer Chain Elongation of Poly( $N$-Isopropylacrylamide) and Poly(Acrylamide) by Atomic Force Microscopy. Journal of Physical Chemistry B, 104, 10258-10264. https://doi.org/10.1021/jp000459f

[18] Taninaka, A., Hirano, Y., Takeuchi, O. and Shigekawa, H. (2012) Force Measurement Enabling Precise Analysis by Dynamic Force Spectroscopy. International Journal of Molecular Sciences, 13, 453-465. https://doi.org/10.3390/ijms13010453

[19] Kuhn, M., Janovjak, H., Hubain, M. and Muller, D.J. (2005) Automated Alignment and Pattern Recognition of Single-Molecule Force Spectroscopy Data. Journal of Microscopy, 218, 125-132. https://doi.org/10.1111/j.1365-2818.2005.01478.x

[20] Kasas, S., Riederer, B.M., Catsicas, S., Cappella, B. and Dietler, G. (2000) Fuzzy Logic Algorithm to Extract Specific Interaction Forces from Atomic Force Microscopy Data. Review of Scientific Instruments, 71, 2082-2086. https://doi.org/10.1063/1.1150583

[21] Chtcheglova, L.A. and Dietler, G. (2003) Force Spectroscopy of Polyclonal and Monoclonal Anti-Bovine Serum Albumin Antibodies-BSA Complexes. Acta Physica Polonica A, 104, 321-326. https://doi.org/10.12693/APhysPolA.104.321

[22] Yersin, A., Hirling, H., Steiner, P., Magnin, S., Regazzi, R., Huni, B., Huguenot, P., De los Rios, P., Dietler, G., Catsicas, S. and Kasas, S. (2003) Interactions between Synaptic Vesicle Fusion Proteins Explored by Atomic Force Microscopy. Proceedings of the National Academy of Sciences of the United States of America, 100, 8736-8741. https://doi.org/10.1073/pnas.1533137100

[23] Chtcheglova, L.A., Shubeita, G.T., Sekatskii, S.K. and Dietler, G. (2004) Force Spectroscopy with a Small Dithering of AFM Tip: A Method of Direct and Continuous Measurement of the Spring Constant of Single Molecules and Molecular Complexes. Biophysical Journal, 86, 1177-1184. https://doi.org/10.1016/S0006-3495(04)74192-2

[24] Cueille, N., Blanc, C.T., Popa-Nita, S., Kasas, S., Catsicas, S., Dietler, G. and Riederer, B.M. (2007) Characterization of MAP1B Heavy Chain Interaction with Actin. Brain Research Bulletin, 71, 610-618. https://doi.org/10.1016/j.brainresbull.2006.12.003

[25] Hull, J.R., Tamura, G.S. and Castner, D.G. (2007) Structure and Reactivity of Adsorbed Fibronectin Films on Mica. Biophysical Journal, 93, 2852-2860. https://doi.org/10.1529/biophysj.107.109819

[26] Yersin, A., Hirling, H., Kasas, S., Roduit, C., Kulangara, K., Dietler, G., Lafont, F., Catsicas, S. and Steiner, P. (2007) Elastic Properties of the Cell Surface and Trafficking of Single AMPA Receptors in Living Hippocampal Neurons. Biophysical Journal, 92, 4482-4489. https://doi.org/10.1529/biophysj.106.092742 
[27] Chtcheglova, L.A., Haeberli, A. and Dietler, G. (2008) Force Spectroscopy of the Fibrin(ogen)-Fibrinogen Interaction. Biopolymers, 89, 292-301. https://doi.org/10.1002/bip.20910

[28] Denisov, N.N., Shcheglova, L.A., Sekatskii, S.K., Dietler, G. and Nadtochenko, V.A. (2008) Single-Molecule Force Spectroscopy of a Protein Globule Covalently Bound to a Calcite Surface. Russian Journal of Physical Chemistry B, 2, 350-353. https://doi.org/10.1134/S1990793108030032

[29] Hull, J.R., Tamura, G.S. and Castner, D.G. (2008) Interactions of the Streptococcal C5a Peptidase with Human Fibronectin. Acta Biomaterialia, 4, 504-513. https://doi.org/10.1016/j.actbio.2008.01.009

[30] Roduit, C., van der Goot, F.G., De Los Rios, P., Yersin, A., Steiner, P., Dietler, G., Catsicas, S., Lafont, F. and Kasas, S. (2008) Elastic Membrane Heterogeneity of Living Cells Revealed by Stiff Nanoscale Membrane Domains. Biophysical Journal, 94, 1521-1532. https://doi.org/10.1529/biophysj.107.112862

[31] Sekiguchi, H., Hidaka, A., Shiga, Y., Ikai, A. and Osada, T. (2009) High-Sensitivity Detection of Proteins Using Gel Electrophoresis and Atomic Force Microscopy. UItramicroscopy, 109, 916-922. https://doi.org/10.1016/j.ultramic.2009.03.010

[32] Yan, C., Yersin, A., Afrin, R., Sekiguchi, H. and Ikai, A. (2009) Single Molecular Dynamic Interactions between Glycophorin A and Lectin as Probed by Atomic Force Microscopy. Biophysical Chemistry, 144, 72-77. https://doi.org/10.1016/j.bpc.2009.06.009

[33] Zaitsev, B.N., Benedetti, F., Mikhaylov, A.G., Korneev, D.V., Sekatskii, S.K., Karakouz, T., Belavin, P.A., Netesova, N.A., Protopopova, E.V., Konovalova, S.N., Dietler, G. and Loktev, V.B. (2014) Force-Induced Globule-Coil Transition in Laminin Binding Protein and Its Role for Viral-Cell Membrane Fusion. Journal of Molecular Recognition, 27, 727-738. https://doi.org/10.1002/jmr.2399

[34] Hutter, J.L. and Bechhoefer, J. (1993) Calibration of Atomic-Force Microscope Tips. Review of Scientific Instruments, 64, 1868-1873. https://doi.org/10.1063/1.1143970

[35] Butt, H.J., Cappella, B. and Kappl, M. (2005) Force Measurements with the Atomic Force Microscope: Technique, Interpretation and Applications. Surface Science Reports, 59, 1-152. https://doi.org/10.1016/j.surfrep.2005.08.003 